\title{
On the performance of biological movement detectors and ideal velocity sensors in the context of optomotor course stabilization
}

\author{
ANNE-KATHRIN WARZECHA AND MARTIN EGELHAAF \\ Centre for Visual Sciences, Research School of Biological Sciences, Australian National University, GPO Box 475, \\ Canberra ACT 2601, Australia
}

(Received March 17, 1997; ACCEPTed July 30, 1997)

\begin{abstract}
It is often assumed that the ultimate goal of a motion-detection system is to faithfully represent the time-dependent velocity of a moving stimulus. This assumption, however, may be an arbitrary standard since the requirements for a motion-detection system depend on the task that is to be solved. In the context of optomotor course stabilization, the performance of a motion-sensitive neuron in the fly's optomotor pathway and of a hypothetical velocity sensor are compared for stimuli as are characteristic of a normal behavioral situation in which the actions and reactions of the animal directly affect its visual input. On average, tethered flies flying in a flight simulator are able to compensate to a large extent the retinal image displacements as are induced by an external disturbance of their flight course. The retinal image motion experienced by the fly under these behavioral closed-loop conditions was replayed in subsequent electrophysiological experiments to the animal while the activity of an identified neuron in the motion pathway was recorded. The velocity fluctuations as well as the corresponding neuronal signals were analyzed with a statistical approach taken from signal-detection theory. An observer scrutinizing either signal performs almost equally well in detecting the external disturbance.
\end{abstract}

Keywords: Optomotor response, Motion vision, Stability of gaze, Reliability

\section{Introduction}

The extraction of motion information from the continually changing retinal images is one of the most fundamental tasks for many animals including man but also for artificial vision systems. On the basis of behavioral and electrophysiological studies, on the one hand, as well as by computational approaches, on the other hand, several types of elementary motion-detection mechanisms have been proposed (for review, see Borst \& Egelhaaf, 1993; Hildreth \& Koch, 1987). Usually, the usefulness of a particular motiondetection mechanism is assessed on the basis of how faithfully it is able to represent the velocity of a moving stimulus. This standard, however, may be rather artificial and does not necessarily reflect the needs of a particular motion information-processing system. For instance, a vital task of any driver of a car is to avoid crashing into an obstacle and an important task of flying animals is to stabilize their course against disturbances like a gust of wind. In both cases, visual motion cues are decisive for solving the task. However, it is not self-evident and needs to be analyzed whether an explicit representation of velocity is required somewhere in the brain to accomplish such a task. Biological motion detectors gen-

Reprint requests to: Anne-Kathrin Warzecha, Lehrstuhl für Neurobiologie, Fakultät für Biologie, Universität Bielefeld, Postfach 1001 31, 33501 Bielefeld, Germany. E-mail: ak.warzecha@biologie.uni-bielefeld.de erally do not signal the velocity of a moving pattern unambiguously. Rather they confound pattern parameters, such as contrast or texture, with the velocity of the moving stimulus (for review, see Egelhaaf \& Borst, 1993b; and see below). Therefore, biological motion detectors are often thought to perform worse in mediating the above-mentioned tasks than hypothetical devices that signal the velocity of a motion stimulus unequivocally. In the context of optomotor course stabilization, this assumption will be explicitly tested in the present study.

The stabilization of the gaze or the path of locomotion against involuntary deviations is of general importance for many moving animals including humans. The nervous system accomplishes this task by evaluating changes in the retinal image motion induced by disturbances and by exploiting this information to mediate corrective movements of the eyes, the head, or the whole body (Miles \& Wallman, 1993). In this behavioral context, we compare the performance of biological motion detectors with the performance of hypothetical velocity sensors. The experimental investigation is performed on the visual system of the blowfly, because this model system is well established for the analysis of motion information processing and well amenable at both the behavioral and the neuronal level (Reichardt \& Poggio, 1976; Egelhaaf et al., 1988; Hausen \& Egelhaaf, 1989; Egelhaaf \& Borst, 1993a). In most studies, the performance of biological motion-detection systems is assessed by determining motion-dependent neuronal or behavioral 
responses to motion stimuli exclusively defined by the experimenter. However, in a normal behavioral situation the animal operates under closed-loop conditions where the retinal input is set to a large extent by the animal's own actions and reactions rather than by an external source. Hence, the dynamics of natural motion stimuli may differ considerably from those used in most experimental paradigms. Therefore, to approximate a normal behavioral situation, a motion-sensitive neuron in the fly's motion pathway, the H1-cell (Eckert, 1980; Hausen, 1981), is confronted in electrophysiological experiments with motion traces which were generated by the actions and reactions of flies in preceding behavioral closed-loop experiments done in a flight simulator. This experimental approach of replaying motion traces previously generated by the animal itself was pioneered by Heisenberg and Wolf (1988) at the behavioral level and is extended here to include also the neuronal level.

Since in real life animals, in general, do not have the opportunity to look at a given stimulus many times before taking a behavioral decision, responses averaged over many stimulus presentations do not allow assessment of the real-time performance of the system under consideration. Therefore a statistical approach derived from signal-detection theory (Green \& Swets, 1974) was employed. This approach allows assessment of the reliability with which two stimuli can be discriminated on a trial-to-trial basis. The discriminability cannot be assessed with common statistical procedures like, for instance, the " $t$-test." Such tests can only reveal whether different stimuli lead on average to different responses, with a large number of measurements allowing to assess even small differences in mean values as significant. The statistical analysis used in the present study is formally equivalent to the so-called ROC-analysis which is widely employed to evaluate the statistical reliability of neuronal signals and to link neuronal and psychophysical performance (e.g. Cohn et al., 1975; Tolhurst et al., 1983; Bradley et al., 1987; Newsome et al., 1989; Vogels \& Orban, 1990). This analysis conceptually leans on psychophysical discrimination tests in which a subject is confronted with one of two alternative stimuli and has to decide which one has been presented ("two-alternative-forced-choice"). Of course, it is not meant to imply that this kind of decision procedure is implemented somewhere in the nervous system of the fly. Instead, the procedure is just used as a statistical test to assess how reliably two stimuli can be discriminated. It will be shown that a motion-sensitive neuron in the optomotor pathway of the fly performs virtually as well as a hypothetical perfect velocity sensor in detecting an externally imposed disturbance in behaviorally generated motion stimuli. This result has been obtained despite the fact that, in contrast to the hypothetical velocity sensor, the neuronal responses are affected by considerable stochastic signal fluctuations, i.e. neuronal noise.

\section{Material and methods}

Experiments were performed with female blowflies (Lucilia cuprina, Calliphoridae) obtained from laboratory stocks (C.S.I.R.O., Division of Entomology, Canberra).

\section{Behavioral experiments}

For behavioral experiments flies were prepared as described previously (Fermi \& Reichardt, 1963). The head of the animal was fixed to the thorax with wax under light carbon dioxide anesthesia. A triangular piece of cardboard was glued to the wax above the frontal part of the thorax. With the cardboard triangle flying flies were suspended from a torque compensator. This device prevented both rotatory and translatory movements of the animal while it measured the yaw torque in stationary flight (Fermi \& Reichardt, 1963; Götz, 1964). The system was operated under closed-loop conditions, i.e. the turning responses of the fly were fed back to the animal's visual input in a similar way as in a free-flight situation. Hence, an intended turn of the fly in one direction induced a displacement of a stimulus pattern in the opposite direction. In this way, the visual input was affected by the fly's own actions and reactions.

Two CRT screens (Tektronix 608, Wilsonville, OR) were used for visual stimulation. The stimulus pattern was generated at a frame rate of $183 \mathrm{~Hz}$ by an image synthesizer (Picasso, Innisfree, Cambridge, MA). Screens were placed symmetrically at an angle of $45 \mathrm{deg}$ and $-45 \mathrm{deg}$ with respect to the long axis of the fly and perpendicular to the horizontal plane of the animal. The centers of the screens were viewed by the fly at an angular horizontal position of $55 \mathrm{deg}$ or $-55 \mathrm{deg}$ (right and left monitor, respectively) with respect to the fly's frontal midline and at an angular vertical position of 0 deg with respect to the equatorial plane of the fly's eye. The horizontal and vertical extent of the screens amounted to approximately $70 \mathrm{deg}$ and $55 \mathrm{deg}$, respectively. The stimulus consisted of a vertical square-wave grating with 5 periods per screen. The mean luminance was $44 \mathrm{~cd} / \mathrm{m}^{2}$, the contrast 0.92 .

As in previous studies (Reichardt \& Poggio, 1976; Heisenberg $\&$ Wolf, 1984), the velocity of image motion was controlled in such a way that it was proportional to the fly's yaw torque. This was achieved by sampling the torque signal by a 12-bit analogto-digital converter of an I/O-card (DT 2801 A, Data Translation, Marlboro, MA) and by calculating the corresponding displacement of the visual surround. At any time step one byte was written to the digital output port of the I/O-card and fed into the phase input of the image synthesizer. In this way, the position of the stimulus pattern was controlled. Since the image synthesizer has a 7-bit resolution for the spatial phase, it permitted gratings to be displayed at 128 distinct phases. In these closed-loop experiments, the I/O-card had to be switched constantly between data acquisition (torque) and output of data (stimulus position). This was done at a frequency of $400 \mathrm{~Hz}$. The torque signals were stored in the memory of the computer for later data analysis and for replay of the same stimulus conditions in electrophysiological experiments.

The consequences of a disturbance of the flight course for the visual input of the animal were simulated by imposing an external motion bias on the velocity signal resulting from the torque response of the tethered flying fly. In an open-loop situation, this bias would have led to a constant motion of the grating with a temporal frequency of $3.1 \mathrm{~Hz}$ corresponding to an angular velocity of about $44 \mathrm{deg} / \mathrm{s}$ in the center of the screen. The exact figure for the angular velocity differs along the horizontal axis of the monitor screen due to perspective distortion.

The fly's behavioral response, i.e. its yaw torque, is related to the angular velocity of the stimulus pattern by the "coupling coefficient." Since the coupling coefficient corresponding to the natural free-flight situation is not known (see Reichardt \& Poggio, 1976; Heisenberg \& Wolf, 1984), a wide range of coupling coefficients were used in preliminary experiments. For all coupling coefficients the results were essentially the same, i.e. the fly could compensate an externally imposed motion signal in a similar way. With the coupling coefficient chosen in the present study a torque of $1 \times 10^{-8} \mathrm{Nm}$ led to an angular velocity of the pattern of $10.8 \mathrm{deg} / \mathrm{s}$. The torque signal was low-pass filtered with a cutoff 
at $5 \mathrm{~Hz}$. This relatively low cutoff frequency was chosen to ensure that it is well below the resonance frequency of the feedback system which lies between 20 and $30 \mathrm{~Hz}$. It was confirmed in control experiments that the principal features of the response, such as the mean slip-speed under closed-loop conditions as well as the existence of considerable fluctuations in the real-time torque responses around the mean level (see below), did not change qualitatively when the cutoff was set to either $10 \mathrm{~Hz}$ or $2 \mathrm{~Hz}$.

Before the experiments, the torque level corresponding to straight flight ("zero torque level") had to be determined. This measure was necessary because, for instance, at the torque compensator flies that are suspended not entirely symmetrically may generate a nonzero torque even if their muscles are activated in the same way as in a straight flight sequence in free flight. To assess the zero torque level, the fly's responses were measured for $1 \mathrm{~min}$ in a closed-loop situation. During this time a motion bias was imposed that alternated every $5 \mathrm{~s}$ between clockwise and counterclockwise rotation of the stimulus pattern. The mean torque response averaged over the whole time interval was taken as the zero torque level. The computer-controlled procedure for the determination of the zero torque level was repeated after every 10 sweeps of data acquisition (see below), i.e. after about $3.5 \mathrm{~min}$. The zero torque level was determined repetitively, because preliminary experiments revealed that some flies do not maintain their mean torque level for a longer period of time. Instead, their mean torque response slowly drifts to other levels.

The experimental protocol consisted of a series of consecutive sweeps of 18.75-s duration during which torque responses were recorded. During the first $7.5 \mathrm{~s}$ and the last $3.75 \mathrm{~s}$ of each sweep, motion of the stimulus pattern was entirely determined by the torque responses of the fly. In between the external motion bias was added for $7.5 \mathrm{~s}$. After the recording time, data were saved on the hard disk of the computer. In successive sweeps the direction of the external motion bias was alternated.

Altogether 1028 sweeps were recorded from 19 flies. The mean time-dependent torque responses and corresponding slip-speed traces of different flies varied quite a lot, ranging from no perceptible compensation of the motion bias to an almost perfect compensation. For the present study, altogether 139 sweeps of five flies were selected for further analysis and as stimuli in subsequent electrophysiological experiments (see below). In these selected sweeps, the flies compensated the external motion bias particularly well, leading, on average, to a small retinal slip-speed. Sweeps were selected because only when the retinal slip-speed is small, there is a problem at all of detecting an imposed motion bias.

\section{Electrophysiological experiments}

The activity of a motion-sensitive, directionally selective spiking neuron in the third visual neuropil of the fly's brain, the H1-cell, was recorded (Eckert, 1980; Hausen, 1981). The animals were dissected following the same procedure as described in detail in a previous paper (Warzecha et al., 1993). Recordings were performed extracellularly with electrolytically sharpened tungsten electrodes. The electrodes were insulated with nail polish and had resistances of about $4 \mathrm{M} \Omega$. As an indifferent electrode, a glass electrode was filled with Ringer solution and inserted into the haemolymph of the head capsule. Recorded signals were amplified with standard electrophysiological equipment. Extracellularly recorded spikes were transformed into pulses of fixed height and duration. For further analysis, the pulses were fed into a 486 PC through an analog-to-digital converter of an I/O-card (DT 2801-A, Data Translation) at a sampling rate of $1.2 \mathrm{kHz}$. After acquisition, the temporal resolution of the neuronal activity was reduced to elementary time bins of $5 \mathrm{~ms}$.

For visual stimulation of the H1-cell, the monitor screens were arranged in exactly the same way as described above for the behavioral experiments. To investigate the responses of the H1-cell to the retinal image displacements obtained in the behavioral closedloop situation, these displacements were replayed as stimuli and the activity of the cell was recorded. The resting activity was measured for $1 \mathrm{~s}$ before the onset of each sweep. The responses of seven cells to altogether six replay sequences of each of the 139 selected sweeps were recorded, i.e. some cells were confronted with all 139 traces while no cell was confronted with any stimulus trace twice. To assess stochastic as well as deterministic fluctuations around the mean response level, two particular sweeps were presented to three cells between 30 and 60 times.

Data acquisition and evaluation were carried out with a 486 PC. The programs were written in ASYST (Keithley Instruments, Taunton, MA).

\section{Data evaluation}

To assess how well the H1-cell is able to detect the external motion bias in the retinal image displacements resulting from the fly's own actions and reactions, we used the concept of an ideal observer. This observer is assumed to look through two time windows at the activity of a neuron. The activity was induced by motion traces which were generated in the corresponding behavioral experiments when there was no external motion bias or in response to a motion bias, respectively (Fig. 1A). However, the ideal observer does not know which window belongs to which stimulus condition. Instead it is the observer's task to carry out this assignment on the basis of the integrated activity within each of the two windows ("integration windows"). The ideal observer is only assumed to know that, on average, the motion bias leads to an increased activity. Therefore, the observer always assigns the window with the higher activity to the external motion bias. It can be shown (Green \& Swets, 1974) that there is no better strategy to detect the bias when the only information available is the integrated activity within each of a pair of integration windows. Depending on the actual spike counts within the two time windows, the choice of the observer can be correct (more spikes in the window during the motion bias), random (same spike count in both windows), or false (less spikes in the window during the motion bias). When this technique is applied to many response traces, the proportion of correct decisions can be determined as a measure of the reliability with which the imposed motion bias can be detected. A value of 0.5 is obtained when the ideal observer assigns the activity within one of the windows equally often to the time before and during the motion bias indicating that the motion bias cannot be detected. A value of 1 indicates perfect detection of the motion bias. It should be noted that the determination of the proportion of correct decisions does not rely on the assumption that the distributions characterizing the variability of the responses are Gaussian. This is important, because for the motion-sensitive neuron analyzed here, the shape of the spike frequency distribution was found to depend on both the activity level of the cell as well as on the size of the integration window within which the spike count was obtained. In particular, for small integration windows spike frequency distributions deviate considerably from Gaussian distributions. 

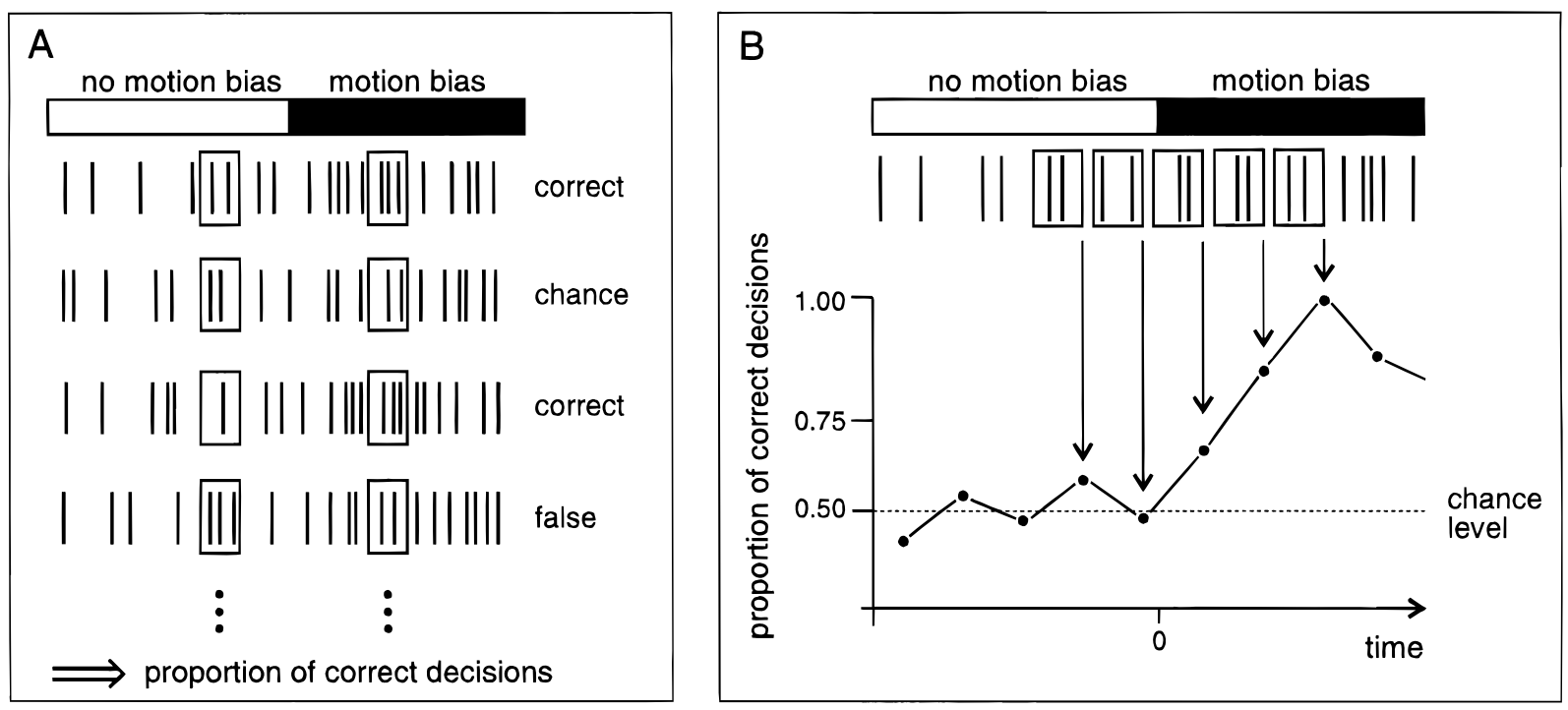

Fig. 1. Procedure to determine the proportion of correct decisions. A: An "ideal observer" looks through two windows at the spike trains that were recorded while the neuron was stimulated with visual motion. The motion trace was generated by a tethered flying fly in a behavioral closed-loop experiment, either before or during the time an external motion bias was added to the retinal image displacements induced by the fly's torque response. In consecutive response traces, the time windows are placed at a fixed position in time relative to the onset of the motion bias. Since, on average, the spike frequency is higher during the motion bias than before it, the observer assigns the window with the higher activity to the time with motion bias. The assignment is correct when a higher activity was recorded during the time with external motion bias than before it (upper panel and third panel from above). Due to neuronal variability, the same number of spikes may occur in both windows rendering the assignment of the observer fortuitous (second panel from above), or the activity may be larger in the window associated with no motion bias leading to a false decision (bottom panel). Applying this procedure to the responses elicited by many motion traces as generated by the fly under behavioral closed-loop conditions without and with external motion bias yields the proportion of correct decisions. B: The proportion of correct decisions is determined as a function of time. One of the two windows through which the observer looks at the spike train is placed at the time interval without a motion bias (reference window, not shown). The activity within the other window ("test window") is compared to the reference activity as illustrated in A (see Material and methods for the exact procedure). The test window is shifted in time steps of $5 \mathrm{~ms}$ across the entire spike train allowing a decision of the observer for every time step. Applying this procedure to many individual responses to identical stimuli yields the proportion of correct decisions as a function of time. For clarity, the integration windows are displayed in the figure slightly smaller than the time step by which they were displaced. Note that for the analysis of the data the windows were considerably larger.

This basic procedure was adapted to the present experimental analysis. Since the mean response amplitude is not constant but depends on the velocity fluctuations of the stimulus trace, the proportion of correct decisions was determined, in extension of the conventional application of this approach, as a function of time (see Fig. 1B). The integrated activity within a test window was compared to the reference activity as determined in an equally sized time window ("reference window") during the time without motion bias. Since it is entirely arbitrary where the reference window should be placed on the response trace during the time without motion bias, the activities within as many neighboring reference windows were taken into account as could be fitted into a time interval of $3.2 \mathrm{~s}$ before the onset of the motion bias (not illustrated in Fig. 1B). The reference windows overlapped partly, each being separated from its neighbors by $5 \mathrm{~ms}$. By using many neighboring reference windows, a better estimate of the reference activity is obtained than by using only a single reference window. The activity within the test window was compared to the activity within each reference window and the proportion of correct decisions was determined from all these comparisons. The test window was shifted in steps of $5 \mathrm{~ms}$ over the entire response trace. For each individual response trace, this procedure leads to the proportion of correct decisions as a function of time. For corresponding elementary time bins, the proportion of correct decisions was averaged over all individual response traces. The proportion of correct decisions was determined for integration windows ranging between 10 and $2800 \mathrm{~ms}$.

To compare the performance of the motion-sensitive $\mathrm{H} 1$-cell with that of a perfect hypothetical velocity sensor, the velocity of the timedependent retinal image displacements, that were generated by the fly in closed-loop experiments and used to stimulate the H1-cell, were subjected to the same statistical procedure as described for the neuron. There were only two modifications. (1) The mean velocity of the stimulus itself within the integration window was determined, instead of the spike count of the neuronal response. (2) The temporal resolution of the velocity trace amounted to $2.5 \mathrm{~ms}$. The test window was therefore compared to reference windows separated by $2.5 \mathrm{~ms}$ instead of $5 \mathrm{~ms}$ and shifted by $2.5 \mathrm{~ms}$ over the velocity trace.

\section{Results}

\section{Real-time performance in a behavioral closed-loop situation}

In the first step of the analysis, the retinal image displacements that are experienced by the fly during optomotor course stabilization were determined in behavioral closed-loop experiments. Fig. 2 

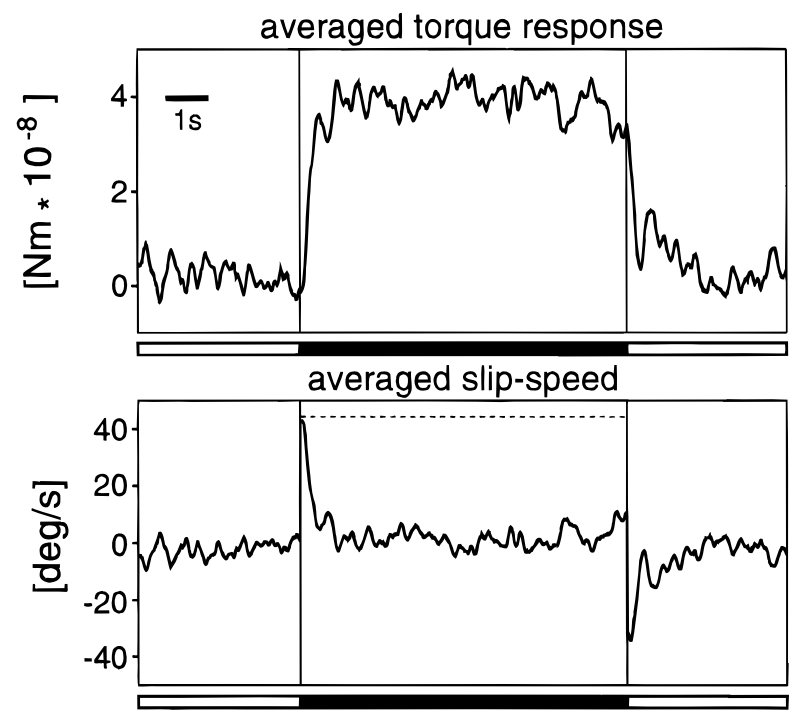

Fig. 2. Mean torque response and corresponding slip-speed in a closedloop situation. During the first and last $3.75 \mathrm{~s}$ of the illustrated time traces the visual input of the fly was controlled exclusively by the torque responses of the animal as is indicated by open bars underneath the diagrams. For a duration of $7.5 \mathrm{~s}$, an external motion bias was added to the pattern displacements induced by the fly's own torque responses as is indicated by the filled bar underneath the diagrams. The dashed line indicates the velocity of the external motion bias under open-loop conditions. Averages are obtained from 139 responses of five flies. (Modified from Warzecha \& Egelhaaf, 1996).

depicts the mean torque response and the corresponding retinal slip-speed before and while flies try to compensate an external disturbance. Without external disturbance flies, on average, generate virtually zero torque that does not lead to a displacement of the stimulus pattern. However, when a motion bias is added, flies generate a torque signal for the duration of the motion bias. This torque leads, after a brief transient phase, to a reduction of the visual consequences of the imposed motion bias with a residual mean slip-speed of less than $10 \%$ of the bias. After cessation of the motion bias flies, on average, stop generating a torque. As a consequence, the mean slip-speed, after a brief transient, approaches zero again.

In contrast to the average performance, the individual torque and slip-speed traces are characterized by large fluctuations around their mean level. Since these fluctuations are very pronounced, it is hardly possible to detect in the slip-speed traces the onset and the offset of the imposed motion bias (see examples in Fig. 3). To quantify the amplitude of the velocity fluctuations, the standard deviation from the mean slip-speed was determined for all selected sweeps. The standard deviation calculated on the basis of $2.5-\mathrm{ms}$ bins during the last $3.2 \mathrm{~s}$ of the motion bias amounts to $35.8 \mathrm{deg} / \mathrm{s}$ as averaged over all sweeps. This figure comes close to the openloop velocity of $44 \mathrm{deg} / \mathrm{s}$ and is almost 10 times larger than the residual average slip-speed under closed-loop conditions.

\section{Responses of a motion-sensitive neuron to motion stimuli generated by the fly's actions and reactions}

How does a motion-sensitive neuron in the optomotor pathway of the fly respond to the strongly modulated motion signals that are
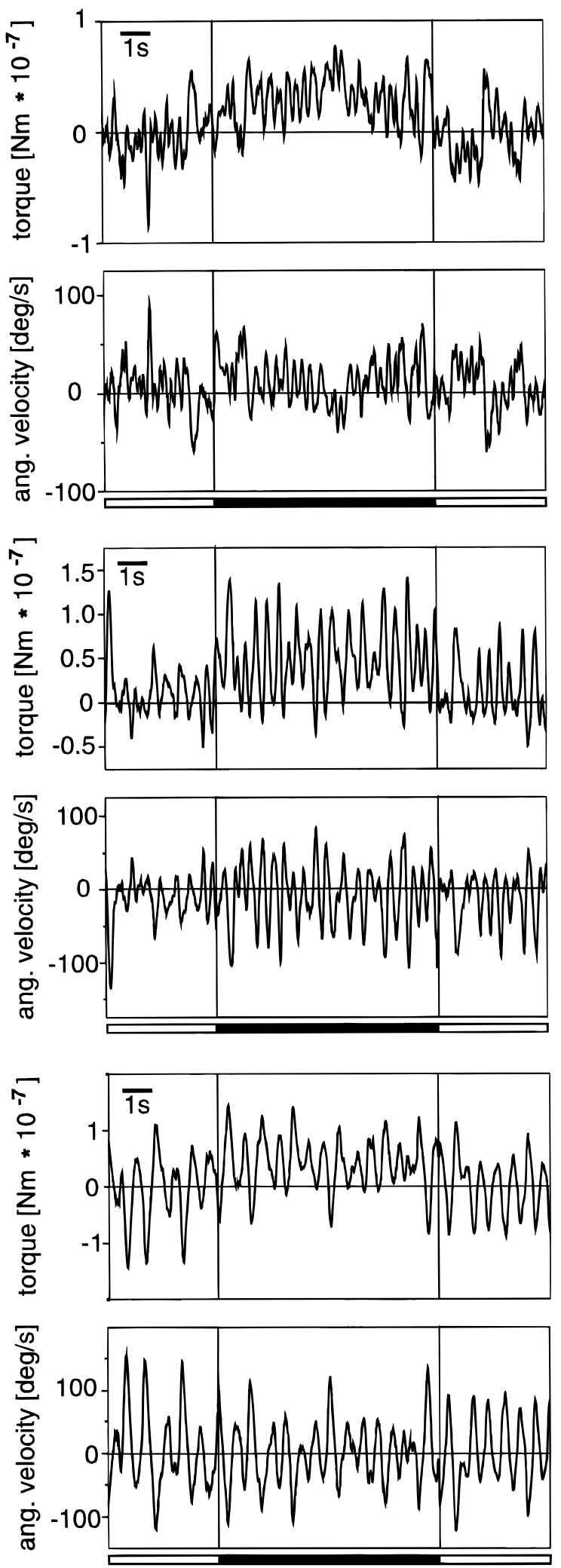

Fig. 3. Three examples of individual torque responses and corresponding slip-speed traces recorded in the same experimental situation as in Fig. 2. The duration of the external motion bias is indicated by the filled bars underneath the diagrams. Open bars indicate the time without external motion bias. The examples originate from three different flies. Note the different ordinate scales. 
generated by the fly's own actions and reactions and how well does the neuron signal the motion bias? To answer this question, the motion traces that were generated by the fly under behavioral closed-loop conditions were replayed while the activity of the H1-cell, a motion-sensitive neuron in the fly's motion pathway (Eckert, 1980; Hausen, 1981) was recorded. This particular neuron was chosen because it can be recorded from longer than most other optomotor neurons. Long recording sessions are requisite to obtain the large amount of data that is necessary for an analysis of the neuronal performance with respect to its reliability. The H1-cell responds directionally selective to motion within large parts of one visual hemisphere due to its input from many retinotopically organized motion-sensitive elements. In this respect it resembles all other optomotor neurons in the fly's brain. The H1-cell is not directly connected to output regions involved in motor-control. It rather is an intrinsic element of the optomotor system and conveys information about wide-field motion to the other half of the brain (Hausen, 1981). In the behavioral experiments both eyes of the fly were stimulated. Hence, equivalent neurons in both halves of the brain were activated which jointly mediate the optomotor turning responses. Therefore, the activity of the two mirror-symmetrical H1-cells, one in each optic lobe, needs to be taken into account. To avoid simultaneous recording from the two heterolateral H1-cells, spike trains of only one cell were recorded while stimulating the animal with two versions of each stimulus trace, one that was identical to the original stimulus of the behavioral situation, the other being inverted so that every displacement of the stimulus pattern occurred in the direction opposite to the original one. In this way the response of the opponent H1-cell in the other half of the brain could be simulated by recording the activity of just one neuron and presenting an additional inverted stimulus trace. In the following analyses spikes were counted as positive signals when the original motion traces were presented; spikes recorded while the inverted version was replayed counted as negative signals. Original and inverted motion traces were always presented consecutively. Neuronal responses to these two stimulus versions were taken as the simultaneous activity of the right and left H1-cell, respectively, assuming that stochastic fluctuations around the mean occur independently in both cells. The replayed stimuli can be divided into two time intervals. During the one interval no motion bias was added in the behavioral situation so that all slip-speed fluctuations are due to the fly's spontaneous torque changes. This interval will be called "time without motion bias." The other stimulus interval represents the motion signal when there was an external motion bias in the corresponding behavioral experiment. This interval will be called "time with motion bias." These terms should not be confounded with a stimulus situation where there is no motion at all or motion with a constant velocity, respectively.

Responses of an H1-cell to an individual motion trace as generated by a fly under behavioral closed-loop conditions and to its inverted counterpart are shown in Fig. 4. Since the H1-neuron is directionally selective, its response is strongly modulated by the profound fluctuations in the velocity of the stimulus that go along with perpetual reversals in the direction of motion. When the opponent spike frequency histograms are subtracted the resulting signal, more or less, follows the slip-speed. Nevertheless, there are characteristic deviations from a linear relationship, indicating that the velocity is not encoded linearly by the H1-neuron. Although the stimulus was the same for each individual response trace, responses are not identical but show some variability. Hence, there are two types of temporal modulations in the responses, (1) deterministic fluctuations that are induced by the fluctuations of the

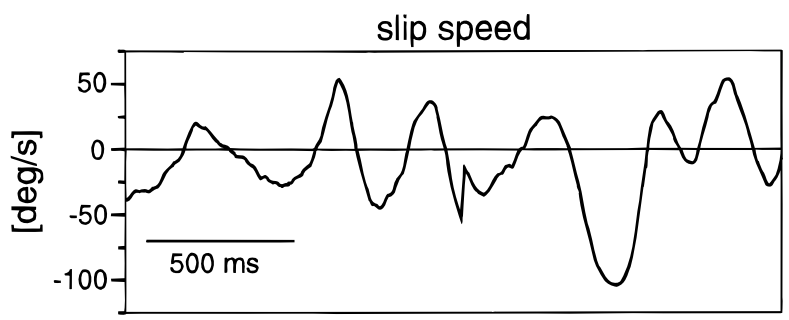

individual neuronal responses

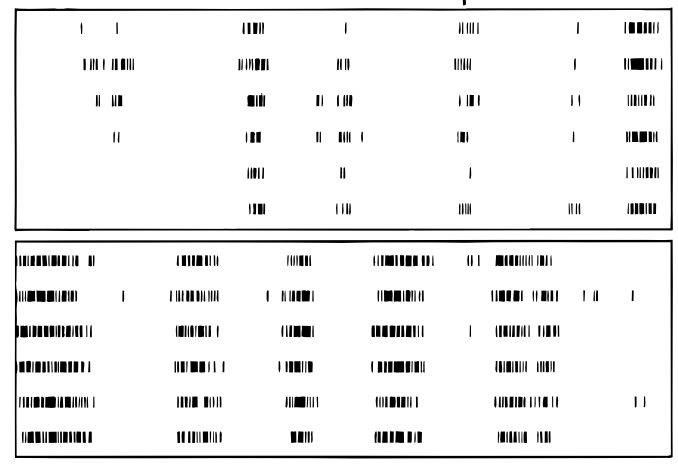

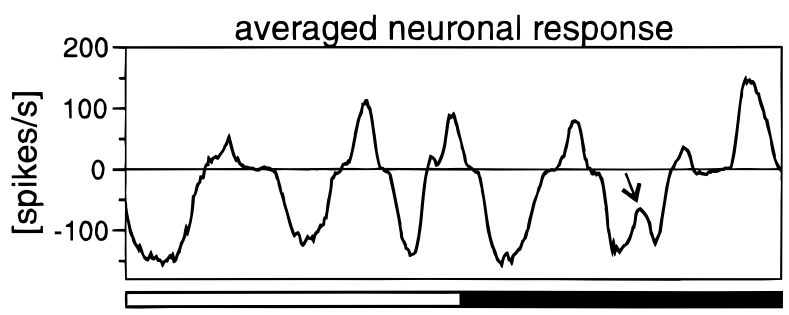

Fig. 4. Responses of an H1-cell in a neuronal replay situation to repetitive stimulation with the same motion trace and its inverted counterpart. The upper diagram depicts the original version of the motion trace. Each of the two middle diagrams shows six consecutive unaveraged responses of the H1cell to identical stimulation either with the original (upper box) or inverted (lower box) motion trace. The occurrence of a spike is indicated by a small vertical line. The spike frequency histogram in the bottom diagram illustrates the difference of $40 \mathrm{H} 1$-cell responses to each of the two opponent stimulus situations. The spike frequency histogram contains the $2 \times 6$ individual response traces shown in the middle diagrams. Note the dent in the time course of the spike frequency histogram marked by an arrow where the mean response obviously does not follow the velocity signal. The filled bar underneath the bottom diagram denotes the duration of the external motion bias; the open bar indicates that the motion stimulus was generated exclusively by the actions and reactions of the fly. Note that the neural responses are displayed at an enlarged scale as compared to the behavioral responses shown in Fig. 3.

moving pattern and (2) stochastic fluctuations that are different in each of the individual response traces and, thus, average out when the same stimulus trace is presented to the cell repeatedly.

\section{Discrimination performance of a pair of motion-sensitive neurons and a perfect velocity sensor}

How well the external motion bias can be detected on the basis of the neuronal response is expected to depend on both the mean neuronal response level during the time with external motion bias as well as on the fluctuations around this mean level. To assess the relative contribution of the stimulus-induced and the stochastic components to the response fluctuations two parameters were cal- 
culated. (1) The standard deviation of the mean time-dependent response from the time-averaged response ("stimulus-induced fluctuations") was determined. (2) Within corresponding time windows of consecutive response traces, the standard deviation of the individual responses from the mean response was evaluated. The standard deviations determined in consecutive time windows were averaged. This average was used as a measure of the stochastic response component ("noise"). Since noise and stimulus-induced response fluctuations can be expected to depend on the size of the time window, within which the neuronal activity is taken into account, both types of modulations were determined using differently sized integration windows (Fig. 5). For a small integration window of $10 \mathrm{~ms}$ the noise amounts to about 0.5 spikes per $10 \mathrm{~ms}$ corresponding to $50 \mathrm{spikes/s.} \mathrm{With} \mathrm{increasing} \mathrm{integration} \mathrm{window,}$ the noise amplitude decreases considerably as a consequence of smoothing out the stochastic signal fluctuations. The stimulusinduced fluctuations are much larger than the noise component for all window sizes tested. The amplitude of the stimulus-induced fluctuations does not change much with increasing window sizes up to $100 \mathrm{~ms}$. Only for larger window sizes does the amplitude of these fluctuations start to decrease. Window sizes above about $100 \mathrm{~ms}$ are large enough to smooth out stimulus-induced fluctuations. Thus, under the present stimulus conditions responses of the H1-cell deviate from a mean value more because of deterministic than of stochastic fluctuations.

To compare the performance of the two opponent H1-cells with that of a perfect velocity sensor, i.e. a hypothetical device that signals the instantaneous velocity of a moving pattern unambiguously without being afflicted by noise, it was investigated how well an ideal observer is able to detect the external motion bias on the basis of either the responses of the two opponent $\mathrm{H} 1$-neurons or the velocity

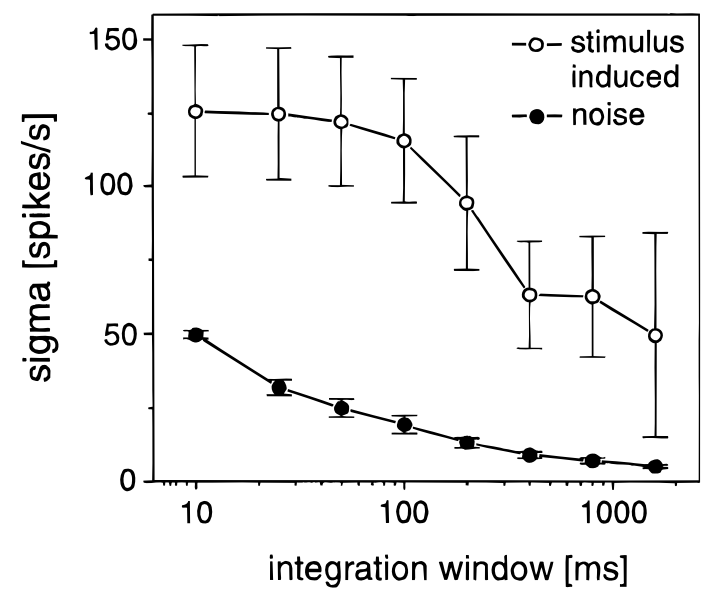

Fig. 5. Stimulus induced deviations (open circles) and noise (filled circles) of the H1-cells' responses in the replay situation for integration windows of variable size. A particular motion trace generated by a fly in a behavioral closed-loop situation and its inverted counterpart were replayed to two H1-cells 30 and 40 times, respectively. A second motion trace and its counterpart were replayed to another H1-cell 60 times. For each cell the noise as well as the motion-induced fluctuations were calculated in neighboring nonoverlapping integration windows as described in the text. The calculation was based on the difference in the responses to the original and inverted version of the motion trace. Starting with the onset of the externally imposed motion bias $3.2 \mathrm{~s}$ of the neuronal response were evaluated. Error bars denote the standard error of the mean associated with the average of the mean values of three cells. traces by which the neuronal responses were induced. The only information available to the observer is the integrated neuronal activity or the mean velocity within a time window ("integration window"). The calculation of the neuronal performance was based on the difference in the responses of the H1-cell to two versions of the motion stimuli, one being the inverted counterpart of the other with respect to its direction of motion. This procedure simulates the simultaneous recording of the H1-neurons in both halves of the brain. Since the size of the integration window is likely to influence the detectability of the motion bias, the proportion of correct decisions was assessed for differently sized integration windows. Small windows enable a reliable detection of the onset of the motion bias for both the neuronal as well as the velocity signals (upper panel, Fig. 6). However, after about $500 \mathrm{~ms}$, when in the behavioral closedloop experiment the fly was able to reduce the motion bias considerably (cf. Fig. 2), small windows do no longer allow detection the motion bias. The proportion of correct decisions then drops to a mean value only slightly above chance level. The performance of the ideal observer on the basis of the velocity signal is very similar to that on the basis of the neuronal response apart from the onset of the motion bias. Here the observer takes slightly longer to achieve a reliability of $75 \%$ when he/she bases the decisions on the responses of the H1-cell rather than on the velocity signal. This difference is due to the fact that the responses of the H1-cell follow the slip-speed with a phase shift of approximately $60 \mathrm{~ms}$ as determined from cross-correlograms (Warzecha \& Egelhaaf, 1996). This time delay has not been corrected for in the diagrams shown in Fig. 6. For an integration window of $800 \mathrm{~ms}$, the proportion of correct decisions rises more slowly after the onset of the external motion bias than for the shorter integration window. The motion bias can be detected with a reliability of more than $75 \%$ as long as the reponse to the onset of the motion bias lies within the integration window (middle panel, Fig. 6). Subsequently, the proportion of correct decisions drops to about $60 \%$. Apart from the time shift at the onset of the motion bias, the decisions of the ideal observer are again about equally reliable no matter whether they are based on the neuronal responses or the velocity signals. The same is true for the largest window used in the present analysis (lower panel, Fig. 6). Here the window is so large that the response to the onset of the motion bias is taken into account for the whole illustrated time interval with motion bias. Consequently, the proportion of correct decisions stays at a relatively high level of slightly more than $75 \%$.

\section{Discussion}

Flying animals are assumed to stabilize their flight path against disturbances such as a gust of wind or asymmetries in the flight motor (for review see Collett et al., 1993). The retinal image displacements that go along with deviations from the flight path are thought to be detected by the optomotor system which then mediates compensatory responses. As a consequence, the retinal error signals get minimized. This means that animals normally operate under so-called closed-loop conditions where the animal's retinal input depends to a large extent on its own actions and reactions. To test how well an external disturbance can be detected under such stimulus conditions, motion traces that were generated by a fly in behavioral responses to an imposed disturbance were presented, on the one hand, in subsequent experiments to a directionally selective motion-sensitive neuron in the fly's optomotor pathway and, on the other hand, to a hypothetical velocity sensor. By employing a statistical approach derived from signal-detection theory, it is shown that the biological motion-detection system performs al- 


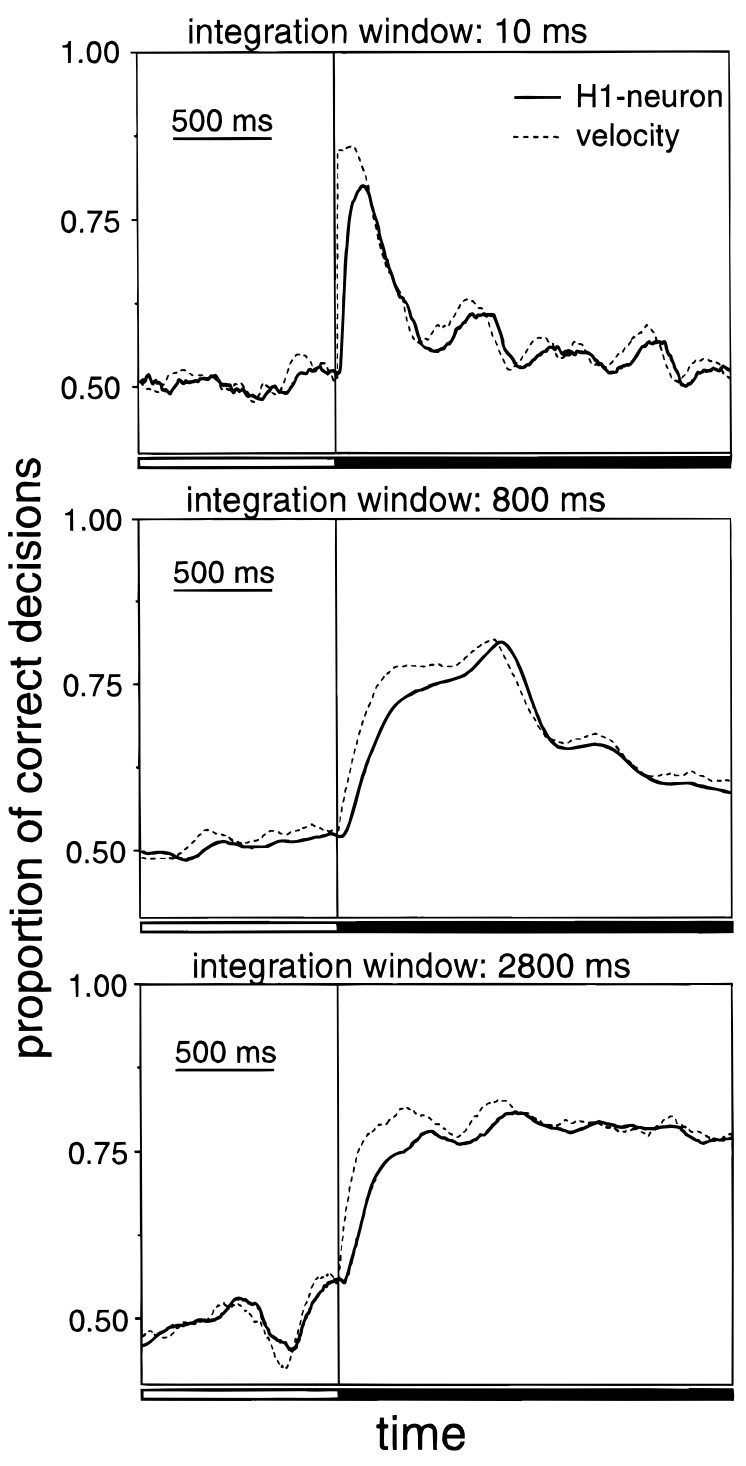

Fig. 6. Time course of the proportion of correct decisions with which an external motion bias can be detected either in the slip-speed traces (dashed line) generated by tethered flying flies under behavioral closed-loop conditions or in the difference between the responses of the H1-cell (solid line) to these motion traces and their inverted counterparts. The filled bars underneath the diagrams denote the duration of the external motion bias; open bars indicate that there was no external motion bias. The proportion of correct decisions was evaluated for integration windows of different lengths as is indicated in the figure. The 139 slip-speed traces recorded in behavioral closed-loop experiments were presented in their original and inverted version to seven cells. Some cells "saw" all $2 \times 139$ traces, some only part of these traces, but no cell "saw" any trace twice. Altogether, the $2 \times 139$ traces were presented six times. The calculation of the neuronal performance was based on the difference in the responses of the H1-cells to two mirror-symmetrical versions of the motion stimuli simulating the simultaneous recording of the H1-neurons in both halves of the brain. To smooth out high-frequency fluctuations in the curves describing the timedependent proportion of correct decisions, the curves were filtered by a running average of $17.5 \mathrm{~ms}$. Note that this procedure does not affect the overall shape of the curves.

most as well in detecting the disturbance in the motion signal as an ideal velocity sensor.

To understand this surprising result, the following questions need to be addressed: (1) What do the behaviorally generated retinal mo- tion traces look like and how do they need to be processed to allow a reliable detection of the external disturbance? (2) What is the functional significance of detecting a motion bias in behaviorally generated stimuli and what are the limitations of the present approach? (3) Why do biological motion detectors perform so well in comparison to an ideal velocity sensor, despite the fact that their responses deviate in a characteristic manner from signalling the velocity of a moving pattern and are concealed by neuronal noise?

What do the behaviorally generated retinal motion traces look like and how do they need to be processed to allow a reliable detection of the external disturbance?

In the behavioral experiments, flies were required to compensate an external motion bias that was constant for some seconds. On average, flies were able to compensate such a disturbance so well that the mean residual retinal slip-speed was rather small compared to the pronounced fluctuations occurring in real-time around this mean level. At least for human observers, these fluctuations render it hardly possible to detect the onset or offset of an external disturbance in the individual motion traces. Nevertheless, flies are able to compensate for such a motion bias astonishingly well and even a single pair of motion-sensitive neurons provides sufficient information to detect the motion bias correctly in approximately $75 \%$ of all presentations. Such a reliable performance is only possible if the neuronal activity is temporally integrated to some extent and, in particular, if the signal at the onset of the motion bias is included in the integration. Hence, when the neuronal activity is evaluated only within a short integration window, the motion bias can be detected reliably only shortly after its onset. Using a larger integration window enables a reliable detection for a longer period of time. Of course, the size of the integration window that is most appropriate to decode an external motion bias in the closed-loop motion traces is likely to depend on the time course of the motion bias. Very large windows are only the most appropriate ones, if the system is required to decode a constant motion bias maintained for a considerable period of time. Here, some kind of temporal integrator following the motion-detection system seems to be necessary to enable a maintained detection of the external disturbance. In the case of a more transient motion bias, smaller integration windows might do much better than larger ones. It should be noted that these qualifications do not affect the main conclusion of the present study. The performance of biological motion detectors and ideal velocity sensors do not much differ with respect to their ability to signal an external motion bias in motion traces generated by the fly under closed-loop conditions.

Two points should be mentioned in the present context. (1) Interestingly, some kind of temporal low-pass filtering with a time constant of some hundreds of milliseconds has been proposed, on the basis of quite different lines of evidence, to play a decisive role in the fly optomotor system (Egelhaaf, 1987; Heisenberg \& Wolf, 1988; Wolf \& Heisenberg, 1990). Hence, there is behavioral evidence that high-frequency fluctuations in retinal image motion are smoothed out before the signals are transformed into the compensatory turning responses. (2) To assess the performance of a neuron in the fly's motion pathway, we did not ask how well the neuronal responses represent the velocity of the retinal image displacements as has been done in a previous study (Bialek et al., 1991). Likewise, we did not ask how well the time course of the stimulusinduced response component can be recovered from the individual response traces, a problem which has been analyzed in a different study (Warzecha \& Egelhaaf, 1997). To accomplish these tasks, the neuronal responses had to be temporally filtered too (Bialek 
et al., 1991; Warzecha \& Egelhaaf, 1997), although with a considerably shorter time constant than was found to be necessary in the present study if not only the rapid transient at the onset of the imposed motion bias is to be detected in the behaviorally generated motion stimuli. Hence, depending on the task the system is required to solve, the neuronal signals need to be processed in a different way.

\section{On the functional significance of the task biological motion detectors and the hypothetical velocity sensor were required to solve and the limitations of the present approach}

In the present account both the biological motion-detection system as well as the hypothetical velocity sensor were required to detect an external disturbance in time-dependent motion signals that were generated beforehand by tethered flies flying in a flight simulator while trying to compensate this external disturbance by their own reactions. Of course, the ability to compensate for disturbances of the flight course imposed by, e.g. a gust of wind or asymmetries in the flight motor, can be assumed to be of vital importance for any flying animal. This ability can also be assumed to rely, at least to a large extent, on the efficient processing of visual cues such as the retinal image displacements that are generated on the eyes as a consequence of external disturbances. Hence, the computational task that the fly motion-detection system, on the one hand, and a hypothetical velocity sensor, on the other hand, were required to solve in the present account is highly significant from a behavioral point of view. Nonetheless, the approach that was used to find out how well different motion-detection schemes accomplish this task needs to be qualified.

The performance of the two motion-detection schemes was tested here in an experimental situation where behaviorally generated motion traces were replayed to a motion-sensitive neuron rather than in a closed-loop situation itself. In this context, it should be emphasized that the real-time performance of a control circuit as is used here to account for optomotor course stabilization can only be expected to be identical under closed-loop and replay conditions, if it does not contain any stochastic element and some kind of nonlinearity such as a saturation characteristic. Otherwise, the performance of the control system may differ for the two conditions, although the retinal input is exactly the same (Warzecha \& Egelhaaf, in preparation). Since the fly's optomotor system exhibits nonlinear behavior as well as stochastic response fluctuations, its replay and closed-loop performance can be expected to differ. Indeed, differences have been found in behavioral closedloop and replay experiments on the fruitfly Drosophila (Heisenberg \& Wolf, 1988; Wolf \& Heisenberg, 1990). At first sight it, therefore, might have been more appropriate to assess the reliability of biological movement detectors and velocity sensors in detecting an external disturbance in the retinal input directly in closedloop rather than in replay experiments. However, in order to compare how well motion-sensitive neurons and hypothetical velocity sensors can detect behaviorally relevant disturbances, one has to present identical stimuli to both systems which is not possible under closedloop conditions. The closest approximation of a closed-loop situation, thus, is to replay stimuli that have been generated under behavioral closed-loop conditions.

\section{How do biological motion detectors relate to perfect velocity sensors?}

The similar performance of a pair of motion-sensitive neurons and of a perfect velocity sensor in detecting an external motion bias in behaviorally relevant motion stimuli is surprising for two reasons. (1) Neuronal responses are affected by stochastic signal fluctuations. (2) The responses of biological movement detectors as inferred from the activity of motion-sensitive neurons as well as from the psychophysical or behavioral performance of humans and animals including the fly deviate in many respects from the responses of perfect velocity sensors (for review, see Borst \& Egelhaaf, 1989; Egelhaaf \& Borst, 1993b).

In many cases, the variance of the stochastic response fluctuations was found to be in the same order of magnitude as the corresponding mean response amplitude (for visual interneurons, see e.g. Tolhurst et al., 1981, 1983; Vogels et al., 1989; Britten et al., 1993). In other studies the neuronal noise was found to be, at least to some extent, considerably smaller than the mean response (Warzecha, 1994; Ruyter van Steveninck et al., 1997; Warzecha et al., 1997). Although there is some discrepancy with respect to the interpretation of these differences, i.e. whether the variability decreases when the stimuli are dynamical rather than constant (Ruyter van Steveninck et al., 1997) or whether the variability decreases at high activity levels of the neuron irrespective of the stimulus dynamics (Warzecha 1994; Warzecha et al., 1997), the standard deviation of the stochastic signal fluctuations as found in the present study was found to be considerably smaller than the fluctuations in the stimulus-induced component of the responses to behaviorally generated stimuli (see Fig. 5). Hence, the stimulus-induced fluctuations of the retinal image displacements elicited by the torque responses of the fly rather than the noise primarily limit the detectability of the motion bias in the individual time-dependent neuronal response traces.

Although at first sight the difference between the output signals of corresponding motion-sensitive neurons in both halves of the brain appears to follow more or less the time-dependent slip-speed signal, at least if averaged over many stimulus presentations, there are characteristic deviations (see also dent marked by arrow in Fig. 4). The time course of the neuronal signals depends, as has been predicted by motion-detection theory, apart from velocity, also on the velocity changes as well as on the higher order temporal derivatives (Egelhaaf \& Reichardt, 1987; Egelhaaf \& Borst, 1989). Nevertheless, these deviations of the neuronal responses from the retinal slip velocity do not much affect the standard deviation of the neuronal activity from its time average and therefore the performance in detecting the motion bias in these signals.

In conclusion, how the performance of a hypothetical velocity sensor and that of biological movement detectors compare strongly depends on the task that is to be solved. If the task is to represent the time course of the retinal velocity, an ideal velocity sensor will obviously be superior to a biological motiondetection system, because the latter is inherently noisy and does not faithfully represent the retinal velocity. However, as was outlined above the optomotor system is not required to represent the velocity but rather to compensate for disturbances of the flight course. In this functional context, a biological motiondetection system does not perform worse than a hypothetical velocity sensor in detecting an external disturbance in motion traces that were generated by the animal itself. Moreover, the characteristic properties of biological motion detectors may even be particularly advantageous, since the decrease in their gain at high pattern velocities prevents the optomotor system from getting unstable when it is to operate with a high feedback gain in order to counteract an external disturbance efficiently (Warzecha \& Egelhaaf, 1996). 


\section{Acknowledgments}

We are most grateful to J. Eikermann for carrying out most of the behavioral and electrophysiological experiments. We thank Volker Dürr, Roland Kern, and Bernd Kimmerle as well as an anonymous referee for their helpful comments on an earlier draft of this paper. During part of this study, A.-K. Warzecha was financed by the Max-Planck-Institut für biologische Kybernetik (Tübingen). This institution also lent part of the equipment used in this study. We thank Professor K. Götz for rendering this support possible.

\section{References}

Bialek, W., Rieke, F., Ruyter van Steveninck, R.R. De \& Warland, D. (1991). Reading a neural code. Science 252, 1854-1857.

Borst, A. \& EgelhaAf, M. (1989). Principles of visual motion detection. Trends in Neurosciences 12, 297-306.

Borst, A. \& EgelhaAf, M. (1993). Detecting visual motion: Theory and models. In Visual Motion and Its Role in the Stabilization of Gaze, ed. Miles, F.A. \& Wallman, J., Amsterdam: Elsevier.

Bradley, A., Skottun, B.C., Ohzawa, I., Sclar, G. \& Freeman, R.D. (1987). Visual orientation and spatial frequency discrimination: A comparison of single neurons and behavior. Journal of Neurophysiology $\mathbf{5 7}$, 755-772.

Britten, K.H., Shadlen, M.N., Newsome, W.T. \& Movshon, J.A. (1993). Responses of neurons in macaque MT to stochastic motion signals. Visual Neuroscience 10, 1157-1169.

Cohn, T.E., Green, D.G. \& Tanner, W.P. (1975). Receiver operating characteristic analysis: Application to the study of quantum fluctuation effects in optic nerve of Rana pipiens.

Collett, T.S., Nalbach, H. \& Wagner, H. (1993). Visual stabilization in arthropods. In Visual Motion and Its Role in the Stabilization of Gaze, ed. Miles, F.A. \& Wallman, J., pp. 239-264. Amsterdam, London, New York: Elsevier.

ECKERT, H. (1980). Functional properties of the H1-neurone in the third optic ganglion of the blowfly, Phaenicia. Journal of Comparative Physiology 135, 29-39.

EgelhaAF, M. (1987). Dynamic properties of two control systems underlying visually guided turning in house-flies. Journal of Comparative Physiology 161, 777-783.

Egelhaaf, M., Hausen, K., Reichardt, W. \& Wehrhahn, C. (1988). Visual course control in flies relies on neuronal computation of object and background motion. Trends in Neurosciences 11, 351-358.

EgelhaAF, M. \& Borst, A. (1989). Transient and steady-state response properties of movement detectors. Journal of the Optical Society of America A 6, 116-127. Errata (1990). Journal of the Optical Society of America A 7, 172.

EgelhaAf, M. \& Borst, A. (1993a). A look into the cockpit of the fly: Visual orientation, algorithms, and identified neurons. The Journal of Neuroscience 13, 4563-4574.

EgelhaAf, M. \& Borst, A. (1993b). Movement detection in arthropods. In Visual Motion and Its Role in the Stabilization of Gaze, ed. WALLMAN, J. \& MiLes, F.A., Amsterdam: Elsevier.

EgelhaAF, M. \& Reichardt, W. (1987). Dynamic response properties of movement detectors: Theoretical analysis and electrophysiological investigation in the visual system of the fly. Biological Cybernetics 56, $69-87$.

Fermi, G. \& ReICHARDT, W. (1963). Optomotorische Reaktionen der Fliege Musca domestica. Abhängigkeit der Reaktion von der Wellenlänge, der Geschwindigkeit, dem Kontrast und der mittleren Leuchtdichte bewegter periodischer Muster. Kybernetik 2, 15-28.
GöтZ, K.G. (1964). Optomotorische Untersuchung des visuellen Systems einiger Augenmutanten der Fruchtfliege Drosophila. Kybernetik 2, 7792.

Green, D.M. \& Swets, J.A. (1974). Signal Detection Theory and Psychophysics. Huntington, New York: Robert Krieger Publishing Co.

HaUsen, K. (1981). Monocular and binocular computation of motion in the lobula plate of the fly. Verhandlungen der Deutschen Zoologischen Gesellschaft 74, 49-70.

Hausen, K. \& EgelhaAf, M. (1989). Neural mechanisms of visual course control in insects. In Facets of Vision, ed. STAVEnGa, D. \& Hardie, R., pp. 391-424. Berlin, Heidelberg, New York: Springer-Verlag.

HeisenberG, M. \& Wolf, R. (1984). Vision in Drosophila. Berlin, Heidelberg, New York, Tokyo: Springer-Verlag.

HeisenberG, M. \& Wolf, R. (1988). Reafferent control of optomotor yaw torque in Drosophila melanogaster. Journal of Comparative Physiology A 163, 373-388.

HildRETH, E.C. \& Koch, C. (1987). The analysis of visual motion: From computational theory to neuronal mechanisms. Annual Review of Neuroscience 10, 477-533.

Miles, F.A. \& Wallman, J. (1993). Visual Motion and Its Role in the Stabilization of Gaze. Amsterdam, London, New York: Elsevier.

Newsome, W.T., Britten, K.H. \& Movshon, J.A. (1989). Neuronal correlates of a perceptual decision. Nature 341, 52-54.

Reichardt, W. \& Poggio, T. (1976). Visual control of orientation behavior in the fly. Part I. A quantitative analysis. Quarterly Review of Biophysics 9, 311-375.

Ruyter van Steveninck, R.R. De, Lewen, G.D., Strong, S.P., Koberle, R., BIALEK, W. (1997). Reproducibility and variability in neural spike trains. Science 275, 1805-1808.

Tolhurst, D.J., Movshon, J.A. \& Thompson, I.D. (1981). The dependence of response amplitude and variance of cat visual cortical neurones on stimulus contrast. Experimental Brain Research 41, 414-419.

Tolmurst, D.J., Movshon, J.A. \& Dean, A.F. (1983). The statistical reliability of signals in single neurons in cat and monkey visual cortex. Vision Research 23, 775-785.

Vogels, R., Spileers, W. \& Orban, G.A. (1989). The response variability of striate cortical neurons in the behaving monkey. Experimental Brain Research 77, 432-436.

Vogels, R. \& Orban, G.A. (1990). How well do response changes of striate neurons signal differences in orientation: A study in the discriminating monkey. Journal of Neuroscience 10, 3543-3558.

Warzecha, A.-K., EgelhaAf, M. \& Borst, A. (1993). Neural circuit tuning fly visual neurons to motion of small objects. I. Dissection of the circuit by pharmacological and photoinactivation techniques. Journal of Neurophysiology 69, 329-339.

WARZECHA, A.-K. (1994). Reliability of neuronal information processing in the motion pathway of the blowflies Calliphora erythrocephala and Lucilia cuprina. Dissertation, Tübingen, Germany.

WARZECHA, A.-K. \& EgELHAAF, M. (1996). Intrinsic properties of biological movement detectors prevent the optomotor control system from getting unstable. Philosophical Transactions of the Royal Society $B$ (London) 351, 1579-1591.

WarZecha, A.-K. \& EgelhaAf, M. (1997). How reliably does a neuron in the optomotor pathway of the fly encode behaviorally relevant information? European Journal of Neuroscience 9, 1365-1374.

Warzecha, A.-K., Kretzberg, J. \& EgelhaAf, M. (1997). Temporal precision of encoding motion of visual interneurons. Current Biology (submitted).

Wolf, R. \& Heisenberg, M. (1990). Visual control of straight flight in Drosophila melanogaster. Journal of Comparative Physiology A 167, 269-283. 